\title{
NEUROFIBROMATOSE TIPO 1: MAIS COMUM E GRAVE DO QUE SE IMAGINA
}

\author{
Juliana Ferreira de Souzaㄹ, luana Lopes de Toledo², Maria Clara Magni Ferreira², Luiz Oswaldo Carneiro Rodrigues ${ }^{3}$, Nilton Alves de Rezende*4
}

Trabalho realizado no Centro de Referência em Neurofibromatose de Minas Gerais - Hospital das Clínicas da Universidade Federal de Minas Gerais, Belo Horizonte, MG

*Correspondência:

Rua Aimorés 462 /116 30.140-070 -

Funcionários, Belo

Horizonte, Minas Gerais

Tel. : 31-32267738

narezende@terra.com.br

\begin{abstract}
RESUMO
OвJETIVo. Determinar a prevalência das características clínicas da neurofibromatose tipo 1 (NF1), avaliar sua gravidade e visibilidade e quantificar baixa estatura, macrocrania, força muscular e as alterações da motricidade oral e da voz nesta enfermidade.

Métodos. 183 pacientes atendidos no Centro de Referência em Neurofibromatose de Minas Gerais (CRNF-MG) foram avaliados quanto à frequência de manifestações clínicas e complicações da NF1. A gravidade e a visibilidade foram avaliadas com o uso de escalas de Riccardi e Ablon respectivamente. As alterações da motricidade oral e da voz foram verificadas com uso do software Vox-Metria, e a medida quantitativa da força muscular realizada com uso de dinamômetro.

Resultados. As manifestações clínicas habituais da NF1 observadas neste estudo são semelhantes às descritas na literatura. Entretanto, mais de $50 \%$ dos pacientes apresentaram gravidade e visibilidade moderada e grave. A incidência de macrocrania e baixa estatura foi maior nos pacientes do CRNF-MG. As alterações da voz e da motricidade oral foram pela primeira vez quantificadas, com rouquidão e distúrbios da motricidade oral observados em mais de $60 \%$ dos pacientes. A força muscular estava reduzida em $67 \%$ dos pacientes.

Conclusão. 0 perfil clínico destes pacientes é semelhante aos relatos prévios da literatura. Mais da metade dos pacientes avaliados apresenta as formas moderada e grave da doença (gravidade e visibilidade), incluindo baixa estatura, macrocrania, distúrbios da voz e da motricidade oral e redução da força muscular. Os resultados desta pesquisa contrariam o conceito tradicional de que a NF1 é uma doença benigna e demonstram alguns aspectos clínicos, ainda não descritos na literatura.
\end{abstract}

UnITERmos: Neurofibromatose 1. Sinais e Sintomas. Gravidade e visibilidade. Força muscular. Distúrbios fonológicos.

\section{INTRODUÇÃO}

Neurofibromatose (NF) é uma denominação genérica para três doenças de origem genética autossômica dominante: neurofibromatose tipo 1 (NF1), neurofibromatose tipo 2 (NF2) e schwannomatose. A NF1 é a doença humana mais frequente causada pelo defeito em um único gene ${ }^{1}$. Apresenta incidência de $1 / 3.000$ nascidos vivos ${ }^{2} e$ é mais frequente que outras doenças como fibrose cística $(1 / 10.000)^{3}$ ou o Diabetes mellitus tipo $1(1 / 13.000)^{4}$. A NF2 e a schwannomatose são mais raras, acometendo cerca de $1 / 25.000$ nascidos vivos ${ }^{5}$.

A primeira publicação de um caso confirmado de NF1 ocorreu em 1768, com a descrição de um paciente com neurofibromas cutâneos e que teria herdado a condição de seu pai ${ }^{6}$. Em 1882, o médico Von Recklinghausen descreveu a patologia de maneira praticamente completa, confirmando a origem nervosa dos tumores e, até hoje, a doença ainda é reconhecida com o seu nome. A NF1 era também reconhecida como doença do "homem elefante", um erro histórico, desde que foi comprovado que o "homem elefante" era portador da Síndrome de Proteus".

A NF1 é herdada de um dos pais em cerca de $50 \%$ dos casos. Os demais pacientes não apresentam história familiar de NF1 sugerindo alta incidência de novas mutações ${ }^{2}$. Ela acomete igualmente ambos os sexos e é herdada de forma autossômica dominante com penetrância completa. 0 gene se localiza no cromossomo $17^{8}$. No entanto, a expressão fenotípica é extremamente variada, inclusive na mesma família, e até mesmo entre gêmeos univitelinos. Várias mutações no gene da NF1 já foram identificadas e estudadas com detalhe, mas não se conseguiu estabelecer uma correlação específica entre o tipo de mutação e

1. Medica do Centro de Referência em Neurofibromatose do HC-UFMG e Mestranda do Programa de Pós-Graduação em Ciências Aplicadas à Saúde do Adulto - Área de Concentração Ciências Clínicas da UFMG, Belo Horizonte, MG

2. Acadêmica de Medicina - Universidade Federal de Minas Gerais e Bolsistas de Iniciação Científica (FAPEMI), Belo Horizonte, MG

3. Doutor em Medicina - Professor Titular da Escola de Educação Física, Fisioterapia e Terapia Ocupacional da UFMG, Belo Horizonte, MG

4. Doutor em Medicina - Professor Associado da Faculdade de Medicina da UFMG, Belo Horizonte, MG 
os diversos fenótipos da NF1. A alteração no gene é responsável pela incapacidade de síntese da proteína neurofibromina, uma proteína citoplasmática que atua como moduladora da atividade de crescimento e diferenciação das células desde a vida intrauterina, e que se expressa primariamente nos neurônios, oligodendrócitos, astrócitos, leucócitos e na medula das suprarrenais ${ }^{9}$.

As principais características clínicas da NF1 são as mancha café-com-leite (MCL), os neurofibromas dérmicos e plexiformes, as falsas efélides axilares e/ou inguinais e os nódulos de Lisch ${ }^{10}$. Trata-se, no entanto, de uma patologia multissistêmica com possibilidade de comprometimento oftalmológico, osteomuscular, cardiovascular, endócrino, do sistema nervoso central e periférico e da aprendizagem ${ }^{11}$. Embora nem todos os pacientes apresentem as complicações clínicas ou estéticas mais graves da doença, a maioria dos pacientes com NF1 e suas famílias sofrem com a incerteza sobre a evolução da doença, o surgimento de novos tumores, o comprometimento estético e a possível transmissão da doença a seus descendentes. Já foi demonstrado que todos estes aspectos geram grande impacto na qualidade de vida dos pacientes e de seus familiares ${ }^{12-14}$.

Em março de 2005, foi criado o Centro de Referência em Neurofibromatose de Minas Gerais (CRNF), no Hospital das Clínicas da UFMG com o objetivo de oferecer uma atenção integrada e multidisciplinar aos pacientes portadores de NF e aos seus familiares, além de promover pesquisa e ensino sobre a doença.

Este trabalho apresenta as características clínicas dos pacientes com NF1 atendidos neste centro comparando-as com os dados da literatura nacional e internacional e descreve alguns aspectos originais desta enfermidade, como o comprometimento da motricidade oral e da voz e a medida quantitativa da força muscular, ainda não descritos.

\section{MÉtodos}

O Projeto de Pesquisa e o respectivo Termo de Consentimento Livre e Esclarecido foram aprovados pelo Comitê de Ética em Pesquisa (COEP) da UFMG. Duzentos e seis prontuários dos pacientes atendidos no CRNF no período de março 2005 a dezembro de 2007 foram transcritos para um protocolo específico. Neste novo protocolo foram incluídos, além de anamnese e heredograma, exame clínico completo, medidas do peso, estatura e perímetro cefálico, resultados de exames complementares, avaliação fonoaudiológica, medida da força muscular e avaliação da gravidade e da visibilidade da doença.

O diagnóstico de NF1 baseou-se no achado de pelo menos dois critérios diagnósticos de acordo com o National Institute of Health (NIH) dos EUA ${ }^{15}$ : seis ou mais manchas café-com-leite, maiores que $0,5 \mathrm{~cm}$ de diâmetro no pré-púbere, e maior que $1,5 \mathrm{~cm}$ de diâmetro no pós-púbere; dois ou mais neurofibromas de qualquer tipo ou 1 neurofibroma plexiforme; efélides em áreas de dobras (regiões axilares e inguinais); displasia da asa do esfenóide ou afilamento da cortical dos ossos longos, com ou sem pseudoartrose; dois ou mais nódulos de Lisch (hamartomas benignos da íris); presença de glioma no nervo óptico e parente de primeiro grau (pai, irmão ou filho) com diagnóstico de NF1. Foram excluídos os pacientes com diagnóstico de NF2 $(n=3)$, aqueles com suspeita de NF1, porém sem critérios suficientes para o diagnóstico $(n=11)$ e pacientes com outros diagnósticos $(n=9)$.
Para avaliação da visibilidade foi utilizada a escala de Ablon $^{12}$ com três níveis de classificação: leve, para pacientes com neurofibromas cutâneos não-visíveis, marcha dentro dos padrões normais e sem repercussões na vida social; moderada, para pacientes com alguns neurofibromas no pescoço, face e mãos, deformidades esqueléticas pouco perceptíveis, e distúrbios do aprendizado; e grave para pacientes com numerosos neurofibromas na face, glioma óptico com deformidade do globo ocular, deformidades esqueléticas evidentes (ameaça a capacidade funcional e a vida).

A gravidade foi avaliada segundo a escala de Riccardi ${ }^{13}$ que classifica os pacientes em quatro categorias: mínima, pacientes que apresentam somente MCL e um número limitado de neurofibromas cutâneos sem repercussão estética ou funcional; leve, para pacientes com grande número de neurofibromas cutâneos, mas de consequências estéticas limitadas e lesões internas assintomáticas; moderada, para aqueles com neurofibromas em grande número (cutâneos e/ou viscerais), pseudoartrose, escoliose, convulsões controladas e grave, onde foram classificados os pacientes com grandes limitações da saúde, cirurgias frequentes, tumores intracranianos e espinhais, schwannomas malignos, neurofibrossarcomas, feocromocitomas, convulsões incontroláveis, retardo mental, hidrocefalia, hipertrofias progressivas.

Para a quantificação da baixa estatura e da macrocrania foram utilizados o perímetro cefálico (PC) e a estatura (E), em centímetros, e foram comparados com as referências internacionais ${ }^{16}$. A estatura foi medida usando-se estadiômetro, e a circunferência frontoccipital foi medida no maior diâmetro sobre a proeminência occipital e sobre o arco das sobrancelhas. Foram considerados como baixa estatura os valores abaixo do percentil 5\% e como macrocrania aqueles acima do percentil 95\%.

Para avaliação específica das alterações da voz e da motricidade oral, 15 pacientes, tiveram a voz gravada e analisada por um programa de computador (VOX-Metria 2.6). Foi solicitado aos pacientes que pronunciassem as vogais /a/, /i/ e /u/ e números na escala de 1 a 10 de forma sustentada e automática, e que dessem sua opinião espontânea a respeito da própria voz. Foram analisadas a frequência, extensão, irregularidade e intensidade da voz. A mobilidade velofaríngea e a função dos órgãos orofaciais foram avaliadas por exame direto.

Para a medida da força muscular, 21 pacientes foram comparados com 21 indivíduos saudáveis, pareados por idade e sexo, e em todos foram feitas três medidas padronizadas da sua força máxima $\left(F_{\max }\right)$ usando dinamômetro manual (0-100 kg Kratos ${ }^{\circledR}$, Brasil). A área da seção transversal foi derivada da medida da circunferência do antebraço (cm) e a força muscular por unidade de área $\left(F_{\text {area }}\right)$ foi calculada $\left(\mathrm{kg} / \mathrm{cm}^{2}\right)^{17}$. A partir das informações coletadas construiu-se um banco de dados, com uso do software SPSS versão 12.0, cuja análise permitiu quantificar a prevalência de manifestações clínicas e complicações da NF1 e avaliar a gravidade e a visibilidade da doença. A significância estatística adotada nos métodos utilizados ( $T$ de Student unicaudal) em todas as variáveis foi o valor de $p<0,05$.

\section{Resultados}

Dos 206 pacientes atendidos no CRNF, 183 preencheram o critério para diagnóstico de NF1 e tiveram seus prontuários avaliados. As frequências das manifestações clínicas 
Tabela 1 - Comparação de frequência (\%) de manifestações clínicas e complicações de NF1 com os resultados de 183 pacientes brasileiros com NF1 atendidos no CRNF-MG

\begin{tabular}{|c|c|c|c|c|c|c|}
\hline Referências & 32 & 33 & 21 & 34 & Total & CRNF-MG \\
\hline Número de pacientes & 1728 & 200 & 55 & 495 & 2478 & 183 \\
\hline Critérios diagnósticos & & & & & Média & \\
\hline Manchas café-com-leite & 90 & 95,5 & 98 & 95 & 94,6 & 97.2 \\
\hline Falsas efélides & 85 & 84 & 94 & 80 & 85,8 & 87.6 \\
\hline Nódulos de Lisch & 59 & 66 & 87,5 & 94 & 76,6 & 78.6 \\
\hline Neurofibromas: a) cutâneos & 54 & 49 & 96 & 89 & 72,0 & 74.3 \\
\hline b) plexiformes & 24 & 25 & 40 & 27 & 29,0 & 34.1 \\
\hline Glioma óptico & 10,7 & 9 & 2 & 10 & 7,9 & 6.3 \\
\hline Displasia óssea da tíbia & 2,2 & 3 & 0 & 3 & 2,1 & 3,5 \\
\hline Displasia óssea do esfenóide & 11,3 & & & 7 & 9,2 & 2,7 \\
\hline \multicolumn{7}{|l|}{ Problemas associados } \\
\hline Cefaléia crônica & & 9 & 45,5 & 22 & 25,5 & 55.5 \\
\hline Escoliose & 26 & 20,5 & 49 & 23 & 29,6 & 30.1 \\
\hline Macrocrania & & 43 & 51 & 38 & 44,0 & 54 \\
\hline Baixa estatura & & 27 & 40 & 18 & 28,3 & 60 \\
\hline Hipertensão arterial & 4 & 3,5 & 11 & 4 & 5,6 & 11 \\
\hline Convulsões & 6,4 & 3,5 & 11 & 5 & 6,5 & 10.2 \\
\hline Pectus excavatum & & 7 & 23 & 11 & 13,7 & 6 \\
\hline Malignização & 5 & 1 & 0 & 5 & 2,8 & 5.2 \\
\hline Fenótipo de Noonan & 3 & 7 & 0 & 9 & 4,8 & 1,6 \\
\hline Puberdade precoce & 4,2 & 1,5 & 0 & 4,2 & 2,5 & 0 \\
\hline \multicolumn{7}{|l|}{ Achados pouco relatados } \\
\hline Assimetria corporal & & & & & & 59 \\
\hline Distúrbios da voz e fala & & & & & & 56.7 \\
\hline
\end{tabular}

e complicações associadas estão resumidas na Tabela 1, e comparadas com as referências citadas na literatura. Setenta e sete pacientes eram do sexo masculino. Pelo menos um parente de primeiro grau estava acometido em $45,2 \%$ dos pacientes, indicando que em $54,8 \%$ dos casos tratava-se de uma nova mutação. A idade variou de 1 a 67 anos, com média de 27 anos.

As MCL foram o achado mais comum (97,2\%), seguido das falsas efélides axilares e/ou inguinais $(87,6 \%)$, nódulos de Lisch (78,6\%), neurofibromas cutâneos (74,3\%) e neurofibromas plexiformes (34,1\%). O glioma óptico foi encontrado em 6,3\% dos pacientes e displasia da tíbia e do esfenóide em 3,5\% e $2,7 \%$ respectivamente. Os problemas clínicos associados mais frequentes foram: assimetrias corporais (59\%), cefaléia crônica (55,5\%), dificuldades de aprendizagem $(54,3 \%)$ e do crescimento $(32,9 \%)$, proptose ocular $(12,9 \%)$, hipertensão arterial $(11,0 \%)$, convulsões $(10,2 \%)$, malignização de neurofibromas plexiformes $(5,2 \%)$. Entre as manifestações ortopédicas a escoliose distrófica foi identificada em 2,9\% dos pacientes, enquanto $27,2 \%$ apresentavam escoliose idiopática. A macrocrania foi observada em 54\% dos pacientes, e baixa estatura em $60 \%$ deles (Figura 1). O fenótipo de Noonan foi identificado em três dos 183 pacientes $(1,6 \%)$.

Os valores da $F_{\max }$ dos homens $(27,1 \pm 14,2 \mathrm{~kg})$ e mulheres com NF1(21,2 \pm . 8,0 kg) foram menores do que esperados para sexo e idade. $67 \%$ dos pacientes com NF1 apresentavam força menor do que seus pares sadios. A média da $F_{\text {area }}$ dos voluntários sadios masculinos $\left(0,82 \pm 0,24 \mathrm{~kg} / \mathrm{cm}^{2}\right)$ foi maior do que dos pacientes com NF1 $\left(0,57 \pm 0,21 \mathrm{~kg} / \mathrm{cm}^{2}\right)$. (Figura 2). Homens sadios mostraram maior $F_{\text {area }}\left(0,97 \pm 0,27 \mathrm{~kg} / \mathrm{cm}^{2}\right)$ do que as mulheres sadias $\left(0,70 \pm 0,15 \mathrm{~kg} / \mathrm{cm}^{2}\right)$ e também do que os pacientes com NF1 masculinos $\left(0,59 \pm 0,25 \mathrm{~kg} / \mathrm{cm}^{2}\right)$. Entretanto, a $F_{\text {area }}$ dos pacientes com NF1 não foi diferente entre os sexos.

Na avaliação da motricidade oral e da voz foram observados: rouquidão (60\%), redução do tempo máximo de fonação (53\%), flutuação na emissão da vogal sustentada (/a/, /i/ e /u/ 86\%), redução da resistência vocal (46\%), hipernasalidade leve (36\%), hipotonia dos órgãos orofaciais (maior que $50 \%$ para todos os órgãos orofaciais) e mobilidade do palato diminuída ou regular $(86 \%)$.

Na avaliação da gravidade com a escala de Riccardi, 43\% dos pacientes foram classificados como de gravidade moderada e $8,1 \%$ foram classificados como graves. Na escala de Ablon para avaliação da visibilidade, $37,6 \%$. dos pacientes foram classificados como moderados e $27,1 \%$ como graves.

\section{Dıscussão}

A grande variabilidade das características clínicas e complicações observadas neste grupo de pacientes brasileiros com diagnóstico de NF1 refletem o acometimento multissistêmico e heterogêneo da NF1, já previamente relatados em alguns trabalhos nacionais e internacionais. 


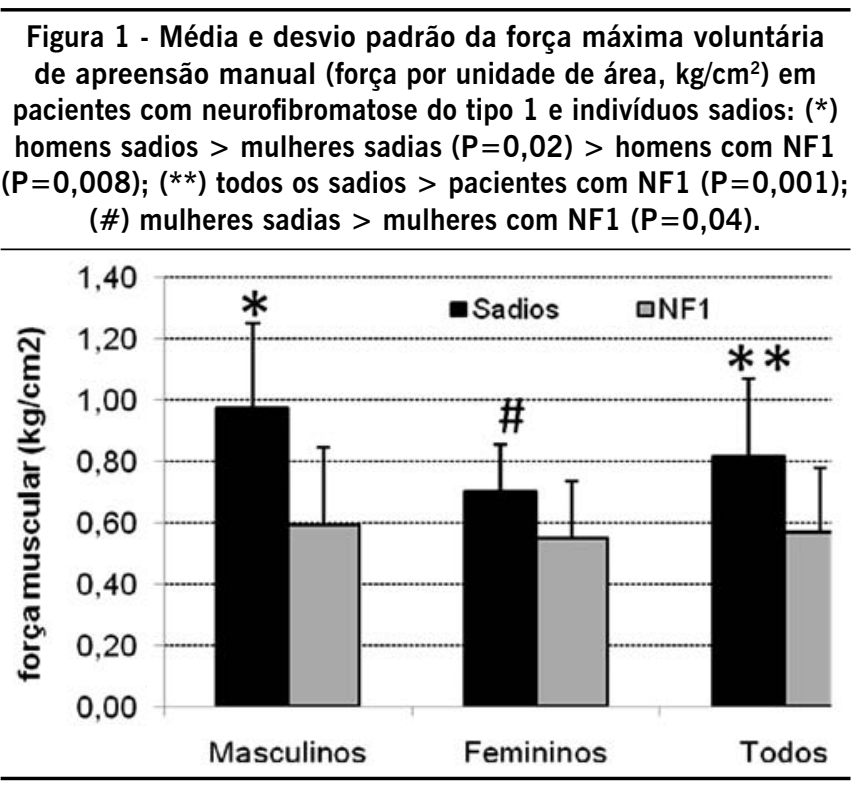
Figura 2 - Prevalência de baixa estatura $(E<)$ e, macrocrania $(P C>)$ associação de $E<$ com $P C>$ em homens e mulheres com neurofibromatose do tipo 1

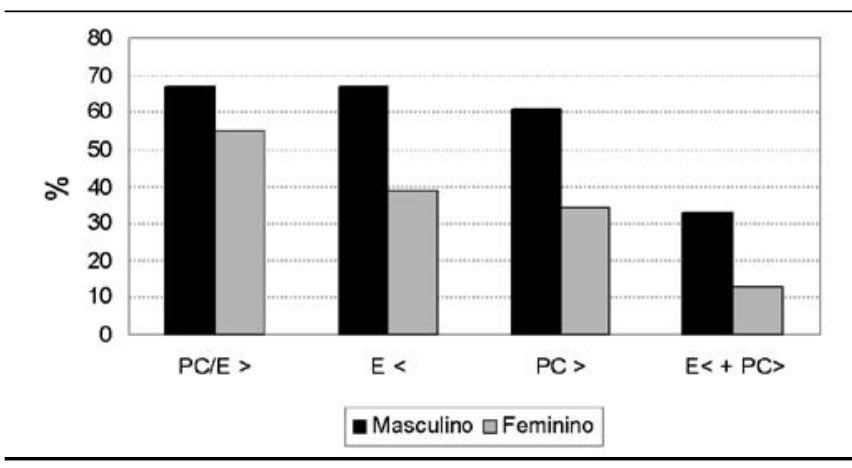

A presença de mais de seis MCL foi a manifestação clínica mais frequente $(97,2 \%)$. Elas estão geralmente presentes ao nascimento ou tornam-se evidentes nos primeiros dois anos de $v_{\text {vida }}{ }^{18}$. Os nódulos de Lisch são hamartomas da superfície da íris, assintomáticos e, quando presentes em número superior a dois, são considerados exclusivos da NF, constituindo critério para confirmação do diagnóstico ${ }^{19}$. Estes nódulos foram observados em $78,6 \%$ dos pacientes, uma frequência menor quando comparada aos $94 \%$ relatados em um dos outros estudos (Tabela 1). É possível que esta menor prevalência em nossa casuística se deva ao fato de que nem todos os nossos pacientes realizaram exame oftalmológico direcionado especificamente para a detecção desta anormalidade.

A prevalência de glioma óptico (6,3\%) é semelhante à observada em outros estudos. O glioma óptico associado à NF1 ocorre em $1 \%$ a $20 \%$ dos pacientes. Destes, aproximadamente $25 \%$ apresentam algum sintoma, como alteração do campo visual, puberdade precoce ou perda da visão. Os gliomas das vias ópticas na NF1 apresentam evolução benigna quando comparado aos gliomas não associados à doença e nos portadores de NF1. Estes tumores raramente causam problemas após a puberdade ${ }^{20}$.

Os neurofibromas plexiformes foram observados em 34,1\% dos pacientes. Esta prevalência é semelhante à descrita em 55 pacientes brasileiros, e um pouco mais elevada do que a descrita na literatura mundial ${ }^{21}$. Outro estudo também realizado no Brasil detectou a prevalência de neurofibromas plexiformes em 26,9\% das 104 crianças e adolescentes avaliados ${ }^{22}$. É possível que esta diferença se deva aos critérios diagnósticos interobservadores. Os neurofibromas plexiformes são tumores primariamente benignos e podem estar associados à hiperpigmentação e/ou hipertricose. Existe uma chance de $7 \%$ a $12,5 \%$ de transformação maligna dos plexiformes para os chamados tumores malignos da bainha dos nervos periféricos, que são altamente agressivos, com surgimento precoce de metástases, de difícil abordagem terapêutica e prognóstico reservado. Nestes tumores é indicada a ressecção cirúrgica complementada por radio ou quimioterapia ${ }^{23}$.

A cefaléia crônica está frequentemente associada à NF1, e a enxaqueca e a cefaléia tensional são os tipos mais comuns. Entretanto, este sintoma pode estar associado com hidrocefalia e/ou lesões expansivas intracranianas em 1,2\% dos casos. Neste estudo, 55,5\% dos pacientes relataram a presença de cefaléia. Não se pode descartar a possibilidade deste sintoma estar relacionado à ansiedade e depressão, e a outros transtornos psicológicos frequentemente observados na NF1 ${ }^{10}$.

Neste grupo foi avaliada a assimetria corporal global, diferentemente de outros estudos que avaliaram apenas assimetria facial. Esta abordagem pode justificar a alta prevalência destas assimetrias (59\%) quando comparada a outros estudos. Além disso, a presença de algumas características clínicas da NF1 como pectus excavatum (6\%) e escoliose idiopática (27,2\%) podem ter contribuído para maior frequência desta alteração. A escoliose é a alteração óssea mais frequente na NF1, presente em cerca de $25 \%$ dos casos, e pode variar de lesões discretas até formas graves. A sua causa ainda não está completamente estabelecida, mas pode estar relacionada à displasia mesodérmica ${ }^{24}$.

A macrocrania afeta grande parte dos indivíduos com NF1. Neste estudo, ela foi observada em $54 \%$ dos pacientes (30/56). Sabe-se que o perímetro cefálico está relacionado com as condições de nutrição, especialmente no primeiro ano de vida ${ }^{25}$, mas na NF1 a hidrocefalia ou a presença de neurofibromas plexiformes na caixa craniana podem determinar um aumento do perímetro cefálico. Parece não haver causa específica para a macrocrania na maioria dos casos $^{13}$.

A prevalência de baixa estatura foi de $60 \%$ (34/56), de acordo com o percentil de $5 \%$ estabelecido, frequência maior que a relatada na literatura ${ }^{26,27}$. As possíveis causas da baixa estatura na NF1 não estão bem estabelecidas. Neste grupo, a baixa estatura pode estar relacionada a problemas nutricionais, mas não se pode descartar que baixos níveis de vitamina $D$ e problemas osteomusculares correlatos, já descritos na NF1, possam contribuir para este achado ${ }^{28}$. Estas especulações devem ser mais bem estudadas.

O distúrbio de voz e motricidade oral foi identificado durante a avaliação clínica e quantificado pela primeira vez como um dos problemas mais frequentemente observados nos 
pacientes portadores de NF1 (56,7\%). As alterações de voz e da motricidade oral ocorrem, provavelmente, em decorrência de alterações neurológicas que ocasionam insuficiência do músculo velofaríngeo e da musculatura orofaríngea. A avaliação fonoaudiológica deve ser realizada em todos os pacientes portadores de NF1. É possível que uma intervenção terapêutica específica e precoce possa contribuir para minimizar estes distúrbios e melhorar o desempenho acadêmico dos pacientes portadores de NF129.

A hipotonia muscular foi observada clinicamente em 32\% dos pacientes. Esta observação motivou a realização de uma análise quantitativa original, para quantificar força muscular. Dos 21 pacientes aleatoriamente avaliados e comparados com controle pareados pelo sexo e idade, constatou-se que $67 \%$ dos portadores de NF1 apresentaram força muscular menor do que indivíduos sadios (Figura 2). Este achado pode estar relacionado com as alterações genéticas da NF1, que resultam em disfunção da coordenação motora nestes pacientes. A possível causa da redução da força muscular nestes pacientes, de origem central ou periférica, precisa ainda ser esclarecida. Estudos neste sentido também estão sendo realizados no CRNF-MG.

É interessante notar que as alterações musculoesqueléticas (baixa estatura, macrocrania e redução da força muscular) parecem afetar predominantemente o sexo masculino. Este achado ainda não foi descrito na literatura, merecendo, portanto, novas investigações, uma vez que se admite, até o momento, que a NF1 afete de forma semelhante ambos os sexos.

$\mathrm{O}$ atendimento dos pacientes com NF1 mostra que a doença apresenta grande impacto na qualidade de vida, tanto do ponto de vista clínico como no aspecto estético. A doença se constitui numa verdadeira agressão à autoimagem e à auto-estima. Afeta a aparência, compromete a aprendizagem e o desempenho no trabalho e, consequentemente, o status econômico. Nesta casuística, 56,7\% dos pacientes relatou algum tipo de dificuldade da aprendizagem, resultado consistente com as frequências previamente relatadas ${ }^{30}$.

A aplicação da escala de classificação da gravidade de Riccardi indicou que a maior parte dos pacientes apresentou as formas moderada e grave da doença $(51,1 \%)$ enquanto na avaliação da visibilidade (escala de Ablon), mais da metade dos pacientes apresentaram a forma moderada ou grave $(64,7 \%)$. Alguns estudos demonstraram que a gravidade clínica e a visibilidade da NF1 têm correlação com a qualidade de vida informada pelos pacientes ${ }^{17,37}$. Considerando estes aspectos e de acordo com os dados obtidos nesta pesquisa, a expressão "benigna" não se aplica à NF1, como tem sido convencionalmente designada.

Apesar da NF1 ser uma doença genética comum5, ela vinha recebendo pouca atenção científica até a década de 90 . Desde então, foi possível o mapeamento genético da doença, avanço no entendimento dos mecanismos envolvidos em sua fisiopatologia e surgimento de possibilidades de intervenção terapêutica específica para algumas das manifestações clínicas desta enfermidade ${ }^{31}$.

\section{Conclusão}

A frequência dos critérios diagnósticos e da maioria das complicações da NF1 entre os pacientes atendidos no CRNF-MG é semelhante à prevalência descrita na literatura. Por outro lado, neste centro, mais da metade dos pacientes apresentaram as formas moderada e grave da doença, incluindo baixa estatura, macrocrania, distúrbios da voz e da motricidade oral e redução da força muscular. Os resultados desta pesquisa contrariam o conceito tradicional de que a neurofibromatose tipo 1 é uma doença benigna.

\section{Agradecimentos}

Este estudo foi apoiado pela Fapemig (APQ-6125-4.01/07)

Conflito de interesse: não há

\section{SUMMARY}

Neurofibromatosis type 1: more fReQuent and SEVERe then USUALLY THOUGHT

OBJECTIVE. To study prevalence of clinical features among Brazilian patients diagnosed with neurofibromatosis type 1(NF1), comparing these features with international data to evaluate the severity and visibility of the disease and quantify less frequent manifestations such as short stature, macrocephaly, muscle strength, voice abnormalities and oral motor disorders.

METHODS. 183 patients diagnosed with NF1, attended at the Neurofibromatosis Outpatient Reference Center, were evaluated for clinical manifestations and complications of NF1. Severity and visibility were verified using the Riccardi and Ablon scales respectively. Voice abnormalities and oral motor disorders were quantified using the Vox-Metria software and maximal voluntary muscle strength (MVMS) was quantified using a handgrip dynamometer.

RESULTS. Clinical manifestations of NF1 observed were comparable to those described in literature. However, more then $50 \%$ of patients presented severity and visibility classified as moderate and severe. The incidence of macrocephaly and short stature was higher among the Brazilian patients. Voice abnormalities and oral motor disorders were quantified for the first time, with hoarseness and oral motor disorders observed in more then $60 \%$ the patients. Maximal voluntary muscle strength was found to be reduced in $67 \%$ of patients.

CONCLUSION. The main clinical features of these patients are similar to those reported in previous studies. More then one-half of the patients presented moderate and severe levels of NF1 (severity and visibility), including short stature, macrocephaly, voice abnormalities and oral motor disorders and decreased muscle strength. These results are in disagreement with the traditional concept that NF1 is a benign disease and also disclosed some clinical aspects not previously reported. [Rev Assoc Med Bras 2009; 55(4): 394-9]

KEY WORDS: Neurofibromatosis type 1. Clinical manifestation. Severity and visibility. Muscular strength. Speech disorders.

\section{REFERÊNCIAS}

1. Huson SM, HarperPS, Compston DA. Von Recklinghausen neurofibromatosis: a clinical and population study in south-east Wales. Brain. 1998;111:1355-8.

2. Friedman, JM. Epidemiology of neurofibromatosis type 1. Am J Med Genet. 1999;89:1-6.

3. Raskin S, Phillips JA 3rd, Krishnamani MRS, Vnencak-Jones C, Parker RA, Rozov T, et al. DNA analysis of cystic fibrosis in Brazil by direct PCR amplification from Guthrie Cards. Am J Med Genet. 1993;46:665-9. 
4. Ferreira SRG, Franco LJ, Vivolo MA, Negrato CA, Simões ACP, Venturelli CR. Population based incidence of IDDM in the state of São Paulo, Brazil. Diabetes Care. 1993; 16:701-4

5. Ferner RE, Huson SN, Thomas N, Moss C, Willshaw H, Evans DG, et al. Guideline for the diagnosis and management of individuals with neurofibromatosis 1. J Med Genet. 2007, 44: 81-8.

6. Arkenside M. Observation on cancer. Trans Med Soc Lond. 1768;1:64-92.

7. Korf BR, Rubestein AE. Neurofibromatosis: a handbook for patients, families and health care professionals. $2^{\text {nd }}$ ed. New York (NY): Thieme Medical Publishier; 2005.

8. Barker D, Wright E, Nguyen K, Cannon L, Fain P, Goldgar D, et al. Gene for von Recklinghausen neurofibromatosis is in the pericentromeric region of chromosome 17. Science. 1987;236(4805):1100-2

9. Datson MM, Scrable H, Nordlund M, Sturbaum AK, Nissen LM, Ratner N The protein product of neurofibromatosis type 1 gene is expressed at highest abundance in neurons, Schwann cells, and oligodendrocytes. Neuron. 1992;8:415-28.

10 North K. Clinical aspects of neurofibromatosis 1. Eur J Paediatr Neurol. 1998;2:223-31.

11. Ruggieri M, Huson SM. The neurofibromatosis: a overview. Ital J Neurol Sci. 1999;20:89-108.

12. Ablon J. Gender responsetoneurofibromatosis 1. SocSciMed.1996;42:99-109.

13. Riccardi VM, Kleiner B. Neurofibromatosis: a neoplastic birth defect with two age peaks of severe problems. Birth Defects Orig Artic Ser. 1977;13:131-8.

14. Sebold CD, Lovell A, Hopkin R, Noll R, Schorry E. Perception of disease severity in adolescents diagnosed with neurofibromatosis type 1. J Adolesc Health. 2004;35:297-302

15. National Institute of Health (NIH). Consensus Development Conference Neurofibromatosis. Arch Neurol. 1988;45:575-8.

16. Hamill PVV, Drizd TA, Johnson CL, Reed RB, Roche AF, Moore WM. Physical growth: national center for health statistics percentiles. Am J Clin Nutr. 1979;32:607-29.

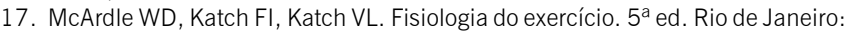
Guanabara Koogan; 2003

18. DeBella K, Szudeck J, Friedman JM. Use of the National Institute of health criteria for diagnosis of neurofibromatosis 1 in children. Pediatrics. 2000;105:608-14.

19. Muniz MP, Ferraz Filho JRL, Souza AS, Zanusso SH, Pavarino-Bertelli EC, Goloni-Bertollo EM. Neurofibromatose tipo 1: aspectos clínicos e radiológicos. Rev Imagem. 2006;28:87-96.

20. Listernick R, Charrow J, Grenwald M, Mets M. Natural history of optic pathway tumors in children with neurofibromatosis type 1: a longitudinal study. J Pediatr. 1994:125:63-6.

21. Trovó-Marques AB, Goloni-Bertollo EM, Valério NI, Pavarino-Bertelli EC, Muniz MP, Teixeira MF, et al. High frequencies of plexiform neurofibromas, mental retardation, learning difficulties, and scoliosis in Brazilian patients with neurofibromatosis type 1. Braz J Med Biol Res. 2005;38:1441-7.
22. Darrigo Jr LG, Gueller M, Bonalumi Filho A, Azulay DR. Prevalence of plexiform neurofibroma in children and adolescents with type 1 neurofibromatosis. J Pediatr. 2007;83:571-3.

23. Evans DG, Baser ME, McGaughran J, Sharif S, Howard E, Moran A. Malignant peripheral nerve sheat tumors in neurofibromatosis type 1. J Med Genet. 2002;39:311-4

24. Muniz MP, Almeida JMR, Aires AS, França FC, Goloni-Bertollo EM. Prevalência de achados radiográficos da neurofibromatose tipo 1: estudo de 82 casos. Radiol Bras. 2002;35:65-70.

25. Macchiaverni LML, Barros Filho AA. Perímetro cefálico: porque medir sempre. Medicina (Ribeirão Preto). 1998;31:595-609.

26. Viana ACS, Liberatore Junior RDR, Goloni-Bertollo EM. Puberdade e crescimento em crianças e adolescentes com neurofibromatose tipo 1. Rev Assoc Med Bras. 2004;50:163-6.

27. Santos, SNMB. Neurofibromatose segmentar cutânea bilateral e estenose da valva pulmonar. [dissertação]. Faculdade de Medicina, Universidade Federal de Minas Gerais - UFMG; 1993.

28. Lammert M, Friedman JM, Roth HJ, Kluwe L, Schooler T, Mautner V-M. Vitamin D deficiency associated with number of neurofibromas in neurofibromatosis 1. J Med Genet. 2006; 43:810-3.

29. Silva CM, Valentin HO, Rodrigues LOC, Rezende NA. High incidence of voice and motor control disturbances in NF1: a preliminary report. In: Models, mechanisms and therapeutic targets. NF Conference 2007: Proceedings of the Childrens Tumor Foundation Conference; 2007 Jun 10-12. Park City, USA; 2007. p.52-3.

30. Page PZ, Page GP, Ecosse E, Korf BR, Leplege A, Wolkestein P. Impact of neurofibromatosis 1 on quality of life: a cross-sectional study of 176 American cases. Am J Med Genet. 2006; 140:1893-8.

31. Acosta MT. Learning disabilities in neurofibromatosis type 1: pharmacological options today and tomorrow. In: Genes to complications to treatment. NF In: Conference 2008: Proceedings of the Childrens Tumor Foundation Conference; 2008 jun 6-10. Bonita Springs, USA; 2008. p. 15.

32. Friedman JM, Birch PH. Type 1 neurofibromatosis: a descriptive analysis of the disorder in 1,728 patients. Am J Med Genet. 1997;70:138-43.

33. North K. Neurofibromatosis type 1: review of the first 200 patients in an Australian clinic. J Child Neurol. 1993;8:395-402.

34. Young H, Hyman S, North K. Neurofibromatosis 1: clinical review and exceptions to the rules. J Child Neurol. 2002;17:613-21.

Artigo recebido: 08/08/08 Aceito para publicação: 19/08/08 